\title{
Numerical Solution of Fractional Electrical Circuits by Haar Wavelet
}

\author{
${ }^{1}$ Sahar Altaf* and ${ }^{2}$ Sumaira Yousuf Khan \\ ${ }^{1}$ College of Humanities and Sciences, \\ Institute of Economics and Technology PAF-KIET, \\ Korangi Creek, Karachi, Pakistan \\ ${ }^{2}$ College of Computer Science and Information Systems \\ Institute of Business Management \\ Korangi Creek, Karachi, Pakistan \\ ${ }^{*}$ Corresponding author: sahar@pafkiet.edu.pk
}

Article history

Received: 2 May 2019

Received in revised form: 8 July 2019

Accepted: 13 August 2019

Published online: 1 December 2019

\begin{abstract}
In this study, numerical approximation of electrical circuits in terms of Caputo fractional time derivative is examined. The order of the derivative being considered is $0 \leq \alpha \leq 1$. Haar wavelet numerical scheme is used to derive the solutions of the fractional electrical circuits, namely RC, LC and RLC. The comparative analysis of numerical simulation of each equation with the classical ones is also provided.
\end{abstract}

Keywords Fractional electrical circuits; Haar wavelet; operational matrix; fractional differential equation

Mathematics Subject Classification 26A33, 34A08, 34K37, 42C40.

\section{Introduction}

The seeds of fractional calculus were planted over 200 years ago, although its application has attracted attention of researchers only in recent decades. Fractional calculus, the derivatives and integrals of non-integer order, is the natural generalization of the classical calculus. It allows better modeling and control of processes of linear and non- linear phenomena that occur in various fields of study. It has become an important tool in many areas of biology, physics, engineering, chemistry, electromagnetism, earth quake study, bioengineering, image processing, and finances etc. [1-5]. In literature, various definitions of fractional derivatives were defined to obtain the solution of fractional differential equations [6-7]. In order to find the solution, several methods are used namely separation of variables [8], finite difference [9], homotopy perturbation [10], and homotopy analysis method [11-12].

Nowadays, wavelet transform and wavelet analysis are attracting considerable attention to find the approximate solutions of ordinary and partial differential equations. It is deeply 
rooted in various disciplines such as deionizing data, image compression, medical science, data compression, signal analysis, solution of initial and boundary value problems and many more. Moreover, wavelets contain numerous significant properties such as compact support, representation of functions at different levels of resolution, orthogonality and representation of polynomials to a specific degree. In recent years, many authors used wavelet techniques in order to solve differential equations. Different types of wavelets exist in literature, such as Haar [13-15], Legendre [16] and Chelyshkov [17]. Haar wavelet is the simplest orthonormal wavelet with compact support and a tool to compute solutions of ordinary and partial differential equations. It was introduced by Alfred Haar [18] in 1910. Based on Haar Wavelet, Chen and Hsiao [19] were the first to propose a method for solving linear systems of ordinary and partial differential equations. Recently many authors applied Haar wavelet method to solve differential equations, some of the important contributions are: Khashan et al. [20] applied Haar wavelet for fractional Riccati type differential equations. Xie et al. [21] proposed Haar wavelet method for approximating the solution of a coupled system of fractional-order integraldifferential equations. Also, Oruc et al. [22] used Haar wavelet method for the approximate solutions of two-dimensional time fractional reaction sub-diffusion equation. Due to simplicity of the proposed method, it is now emerging to be a predominant new technique.

This study is devoted to the application of Haar wavelet in solving a class of electrical circuits RL, LC and RLC, using Caputo fractional derivative. To the best of our knowledge, no attempt has been made in order to solve electrical circuits by using Caputo fractional derivative, hence making our effort a novel approach for solving such problems. However, some recent efforts were made close to our approach but not as simple and efficient, Gomez et al. [23] found the solution of electrical circuits using the same Caputo derivative and applied numerical Laplace transform. Furthermore, they studied RLC circuit in time domain and obtained solution in terms of Mittag-Laffler function. Shah et al. [24] solved RL electrical circuit by using Laplace transform of the fractional derivative in the Caputo sense whereas, Alsaedi et al. [25] discussed fractional electrical circuit equations using fractional calculus and various fractional definitions. Gill et al. [26] derived the solution of RLC circuit using Sumudu transform and got solution in terms of Mittag-Laffler function. Arora and Chauhan [27] applied Legendre wavelet to solve fractional electrical circuits. Atangana et al. [28] examined RLC circuit model via the fractional derivative without singular kernel. Also Gomez et al. [29] worked on finding analytical and numerical solutions of electrical circuits described by fractional derivatives. Recently, Sene et al. [30] derived analytical solutions of electrical circuits considering certain generalized fractional derivatives.

This manuscript has five sections: Some essential definitions of fractional calculus are presented in Section 2, In Section 3, Haar wavelet and its operational matrix are discussed. Section 4 is devoted to Fractional electrical circuit models that are analyzed approximately by Haar wavelet and compared with their classical ones. The conclusion is given in Section 5.

\section{Theory of Fractional Calculus}

There are many definitions related to fractional order, including Riemann-Liouville, Caputo, Riesz and Jumarie's fractional derivative. Here, we list some essential definitions of the fractional calculus used in this paper. 
Definition 1: Suppose $\sigma(x), x>0$, is a function space in space $C_{\mu}, \mu, \in \mathbb{R}$. There exists a number $q$ belongs to $\mathbb{R}, q(>0)$ such that $\sigma(x)=x^{q} g(x)$ where $g(x) \in C[0, \infty)$, which can be written as $C_{\mu}^{m}$ if $F^{(m)} \in C_{\mu}, m \in \mathbb{N}$.

Definition 2: The Reimann-Liouville fractional integral operator $J^{\alpha}$ for order $\alpha$ is defined as

$$
\left\{\begin{array}{l}
J^{\alpha} \sigma(x)=\frac{1}{\Gamma(\alpha)} \int_{0}^{x}(x-t)^{\alpha-1} \sigma(t) d t, \text { when } \alpha>0, \quad x>0, \\
J^{\alpha} \sigma(x)=\sigma(x) .
\end{array}\right.
$$

Some properties are as follows:

i. $J^{\alpha} J^{\beta}=J^{\alpha+\beta}$

ii. $J^{\alpha} J^{\beta}=J^{\beta} J^{\alpha}$

iii. $\left(J^{\alpha} J^{\beta}\right) f(t)=\left(J^{\beta} J^{\alpha} f\right)(t)$

iv. $J^{\alpha}(t-\alpha)^{\varphi}=\frac{\Gamma(\varphi+1)}{\Gamma(\alpha+\varphi+1)}(t-\alpha)^{\alpha+\varphi}$

where $\alpha, \beta \geq 0$ and $\varphi>-1$.

Definition 3: Caputo fractional order derivative $D^{\alpha}$ is defined as

$$
D^{\alpha} \sigma(x)=\frac{1}{\Gamma(n-\alpha)}\left(\frac{d}{d x}\right)^{n} \int_{0}^{x}(x-t)^{n-\alpha-1} \sigma(t) d t,
$$

where $n-1<\alpha \leq n, n \in \mathbb{N}, x>0$.

For the interval $n-1<\alpha \leq n, n \in \mathbb{N}, x>0$ and $f \in C_{\mu}^{n} \geq-1, D^{\alpha} J^{\alpha} \sigma(x)=\sigma(x)$, if $x>0$ and $D^{\alpha} J^{\alpha} \sigma(x)=\sigma(x)-\sum_{k=0}^{n-1} \sigma^{(k)}\left(0^{+}\right) \frac{x^{k}}{k !}, t>0$. For detailed study on fractional derivatives refer to $[1-3]$.

\section{Haar Wavelet}

Haar wavelet is defined as

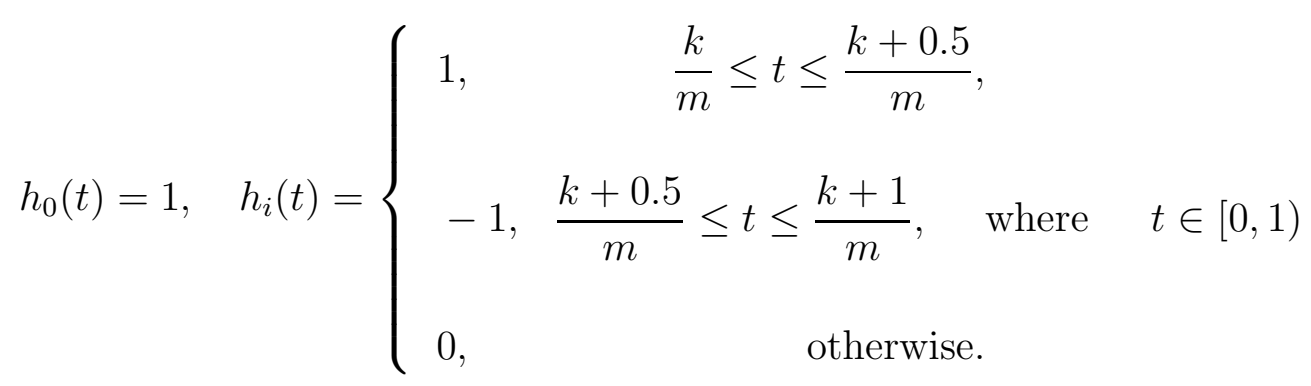

The index $i=0,1,2, \ldots, m-1, m=2^{j}$, where $i=2 j+k-1, j \geq 0$ and $0 \leq k \leq 2^{j-1}$. The maximum value of $i$ is $M=2 m=2^{J+1}$. Note that $j$ and $k$ corresponds to integer decomposition of $i$. 


\subsection{Function Approximation of Haar Wavelet}

Haar wavelet function $w(t)$ can be defined as [31]

$$
w(t)=\sum_{i=0}^{\infty} v_{i} h_{i}(t)
$$

where

$$
v_{i}=\int_{0}^{1} u(t) h_{i}(t) d t .
$$

The function $w(t)$ is piecewise constant in subintervals. So that $w(t)$ resolved at fixed terms

$$
w(t) \approx \sum_{i=0}^{m-1} v_{i} h_{i}(t)=v^{T} H_{m}(t),
$$

where $v=\left[v_{0}, v_{1}, \ldots, v_{m-1}\right]^{T}, H_{m}(t)=\left[h_{0}(t), h_{1}(t), \ldots, h_{m-1}(t)\right]^{T}$. Here $m$ is a power of 2 .

Equation (3) can be written in matrix form as $w=v^{T} H$. Here the vector $v$ is in discrete form and the Haar matrix, $H$ is of order $m=2^{j}$, where $j=0,1,2, \ldots, J$, that is

$$
H=\left[\begin{array}{llll}
h_{0}\left(t_{0}\right) & h_{0}\left(t_{1}\right) & \cdots & h_{0}\left(t_{m-1}\right) \\
h_{1}\left(t_{0}\right) & h_{1}\left(t_{1}\right) & \cdots & h_{1}\left(t_{m-1}\right) \\
\vdots & \vdots & \ddots & \vdots \\
h_{m-1}\left(t_{0}\right) & h_{m-1}\left(t_{1}\right) & \cdots & h_{m-1}\left(t_{m-1}\right)
\end{array}\right] .
$$

To solve the coefficients $v_{i}$, we use wavelet collocation method whose points can be defined as:

$$
t_{i}=\frac{2 i-1}{2 m}, \quad i=1,2, \ldots, m .
$$

Now defining the square Haar matrix of order $m[31]$ :

$$
\eta_{m \times m} \triangleq\left[H_{m}\left(\frac{1}{2 m}\right), H_{m}\left(\frac{3}{2 m}\right), \ldots . H_{m}\left(\frac{2 m-1}{2 m}\right)\right] .
$$

For different values of $m$, we can get the $H$ square matrix. If we set $m=8$, the Haar matrix would be

$$
\eta_{8 x 8}=\left[\begin{array}{cllllccc}
1 & 1 & 1 & 1 & 1 & 1 & 1 & 1 \\
1 & 1 & 1 & 1 & -1 & -1 & -1 & -1 \\
1 & 1 & -1 & -1 & 0 & 0 & 0 & 0 \\
0 & 0 & 0 & 0 & 1 & 1 & -1 & -1 \\
1 & -1 & 0 & 0 & 0 & 0 & 0 & 0 \\
0 & 0 & 1 & -1 & 0 & 0 & 0 & 0 \\
0 & 0 & 0 & 0 & 1 & -1 & 0 & 0 \\
0 & 0 & 0 & 0 & 0 & 0 & 1 & -1
\end{array}\right] .
$$

According to the definition of Haar wavelet, $H$ is known as an orthogonal matrix. 


\subsection{Haar Operational Matrix of Fractional Integration}

Beginning with the operational matrix of Haar wavelet, the first step is to define a set of $m$-term Block Pulse functions as:

$$
\tilde{b}_{i}(t)= \begin{cases}1, & \frac{i}{m} \leq t \leq \frac{(i+1)}{m}, \\ 0, & \text { otherwise }\end{cases}
$$

where $i=0,1,2, \ldots,(m-1)$. The functions $\tilde{b}_{i}(t)$ are orthogonal. That is,

$$
\tilde{b}_{i}(t) \tilde{b}_{l}(t)= \begin{cases}0, & i \neq l, \\ \tilde{b}_{i}(t), & i=l,\end{cases}
$$

where

$$
\int_{0}^{1} \tilde{b}_{i}(\tau) \tilde{b}_{l}(\tau) d \tau=\left\{\begin{array}{lc}
0, & i \neq l \\
1 / m, & i=l .
\end{array}\right.
$$

Since the Haar functions are in the form of piecewise constants, so defining them into $m$-term block pulse functions gives

$$
H_{m}(t)=\eta_{m \times m} \tilde{B}_{m}(t)
$$

where

$$
\tilde{B}_{m}(t) \triangleq\left[\tilde{b}_{0}(t), \tilde{b}_{1}(t), \ldots \tilde{b}_{i}(t) \ldots \tilde{b}_{m-1}(t)\right]^{T}
$$

Kilicman [32] defined Block Pulse operational matrix $\bar{F}^{n}$ as:

$$
\left(I^{n} \tilde{B}_{m}\right)(t) \approx \bar{F}^{n} \tilde{B}_{m}(t),
$$

where

$$
\bar{F}^{n}=\frac{1}{m^{n}} \frac{1}{\Gamma(\alpha+2)}\left[\begin{array}{ccccc}
1 & \xi_{1} & \xi_{2} & \ldots & \xi_{m-1} \\
0 & 1 & \xi_{1} & \ldots & \xi_{m-2} \\
0 & 0 & 1 & \ldots & \xi_{m-3} \\
0 & 0 & 0 & \ddots & \vdots \\
0 & 0 & 0 & 0 & 1
\end{array}\right]
$$

with $\xi_{k}=(k+1)^{n+1}-2 k^{n+1}+(k-1)^{n+1}$.

To construct the Haar operational matrix, let

$$
\left(I^{n} H_{m}\right)(t) \approx P_{m \times m}^{n} H_{m}(t),
$$

where $P_{m \times m}^{n}$ is the square matrix known as the Haar operational matrix. By using equations (10) and (11), we have

$$
\left(I^{n} H_{m}\right)(t) \approx\left(I^{n} \eta_{m \times m} \tilde{B}_{m}\right)(t)=\eta_{m \times m}\left(I^{n} \tilde{B}\right)(t) \approx \eta_{m \times m}\left(I^{n} \bar{F}^{n} \tilde{B}_{m}\right)(t) .
$$

From equations (12) and (13), we get

$$
P_{m \times m}^{n} H_{m}(t)=P_{m \times m}^{n} \eta_{m \times m} \tilde{B}_{m}(t)=\eta_{m \times m} \bar{F}^{n} \tilde{B}_{m}(t) .
$$

The Haar operational matrix $P_{m \times m}^{n}$ is given by

$$
P_{m \times m}^{n}=\eta_{m \times m} \bar{F}^{n} \eta_{m \times m}^{-1} .
$$




\section{Applications}

This section presents the applications of Haar wavelet technique to fractional electrical circuits LC, RC and RLC to find the approximate solution as compared with their classical order solutions.

\section{LC Circuit}

In an LC circuit equation, a combination of charged capacitor and inductor is presented. The fractional order equation of LC circuit is given below:

$$
D^{\alpha} V(t)+\varphi_{0}^{2} V(t)=0, \quad \alpha \in(1,2]
$$

with initial conditions $V(0)=V_{0}$, and $D^{\alpha} V(0)=0$. The classical solution of for $\alpha=2$ is

$$
V(t)_{L C}=V_{0} \cos \left(\varphi_{0} t\right)
$$

where $\varphi_{0}^{2}=\frac{1}{L C}$. To find the approximate solution of equation (16) by Haar wavelet, let

$$
D^{\alpha} V(t)=a_{m}^{T} H_{m}
$$

Integrating equation (18) twice with respect to $t$, we get

$$
V(t)=a_{m}^{T} P^{\alpha} H_{m}+t D^{\alpha} V(0)+V(0) .
$$

After using the initial conditions, we get the result

$$
V(t)=a_{m}^{T} P^{\alpha} H_{m}+V_{0}
$$

Now equation (16) will be transformed to matrix form as

$$
a_{m}^{T} H_{m}+\varphi_{0}^{2}\left[a_{m}^{T} P^{\alpha} H_{m}+V_{0}\right]=0 .
$$

The above matrix equation can be solved by using MATLAB to find the wavelet coefficients vector $a_{m}^{T}$. The numerical solutions of the LC circuit for different values of $\alpha$ are given in Table 1. Also graphical analysis for different values of $\alpha=1.5,1.75,1.99$ with $m=8$ is shown in Figure 1. As it can be clearly seen, for $\alpha=1.99$ with $m=8$, the graphical behavior of fractional LC circuit is similar to the classical solution for $\alpha=2$.

\section{RC Circuit}

Consider the fractional $\mathrm{RC}$ circuit where resistance and charged capacitor are present. The related differential equation of fractional order is

$$
D^{\alpha} U(t)+\mu U(t)=0, \quad \text { where } \quad 0<\alpha \leq 1
$$

with condition $U(0)=U_{0}$ where $\mu=\frac{1}{R C}$. 
Table 1: Numerical Results of LC circuit $\left(L=1, C=1, V_{0}=0.01\right.$ and $\alpha=1.5,1.75,1.99$ and 2$)$.

\begin{tabular}{|c|c|c|c|c|}
\hline \multirow{2}{*}{$t$} & $\alpha=1.5$ & $\alpha=1.75$ & $\alpha=1.99$ & $\alpha=2.0$ \\
\cline { 2 - 5 } & HW & HW & HW & CS \\
\hline $1 / 16$ & $9.8 \times 10^{-3}$ & $9.9 \times 10^{-3}$ & $10.0 \times 10^{-3}$ & $10.0 \times 10^{-3}$ \\
\hline $3 / 16$ & $9.4 \times 10^{-3}$ & $9.6 \times 10^{-3}$ & $9.8 \times 10^{-3}$ & $9.8 \times 10^{-3}$ \\
\hline $5 / 16$ & $8.7 \times 10^{-3}$ & $9.2 \times 10^{-3}$ & $9.5 \times 10^{-3}$ & $9.5 \times 10^{-3}$ \\
\hline $7 / 16$ & $8.0 \times 10^{-3}$ & $8.6 \times 10^{-3}$ & $9.0 \times 10^{-3}$ & $9.1 \times 10^{-3}$ \\
\hline $9 / 16$ & $7.1 \times 10^{-3}$ & $7.8 \times 10^{-3}$ & $8.4 \times 10^{-3}$ & $8.5 \times 10^{-3}$ \\
\hline $11 / 16$ & $6.2 \times 10^{-3}$ & $7.0 \times 10^{-3}$ & $7.7 \times 10^{-3}$ & $7.7 \times 10^{-3}$ \\
\hline $13 / 16$ & $5.3 \times 10^{-3}$ & $6.1 \times 10^{-3}$ & $6.8 \times 10^{-3}$ & $6.9 \times 10^{-3}$ \\
\hline $15 / 16$ & $4.4 \times 10^{-3}$ & $5.1 \times 10^{-3}$ & $5.9 \times 10^{-3}$ & $5.9 \times 10^{-3}$ \\
\hline
\end{tabular}
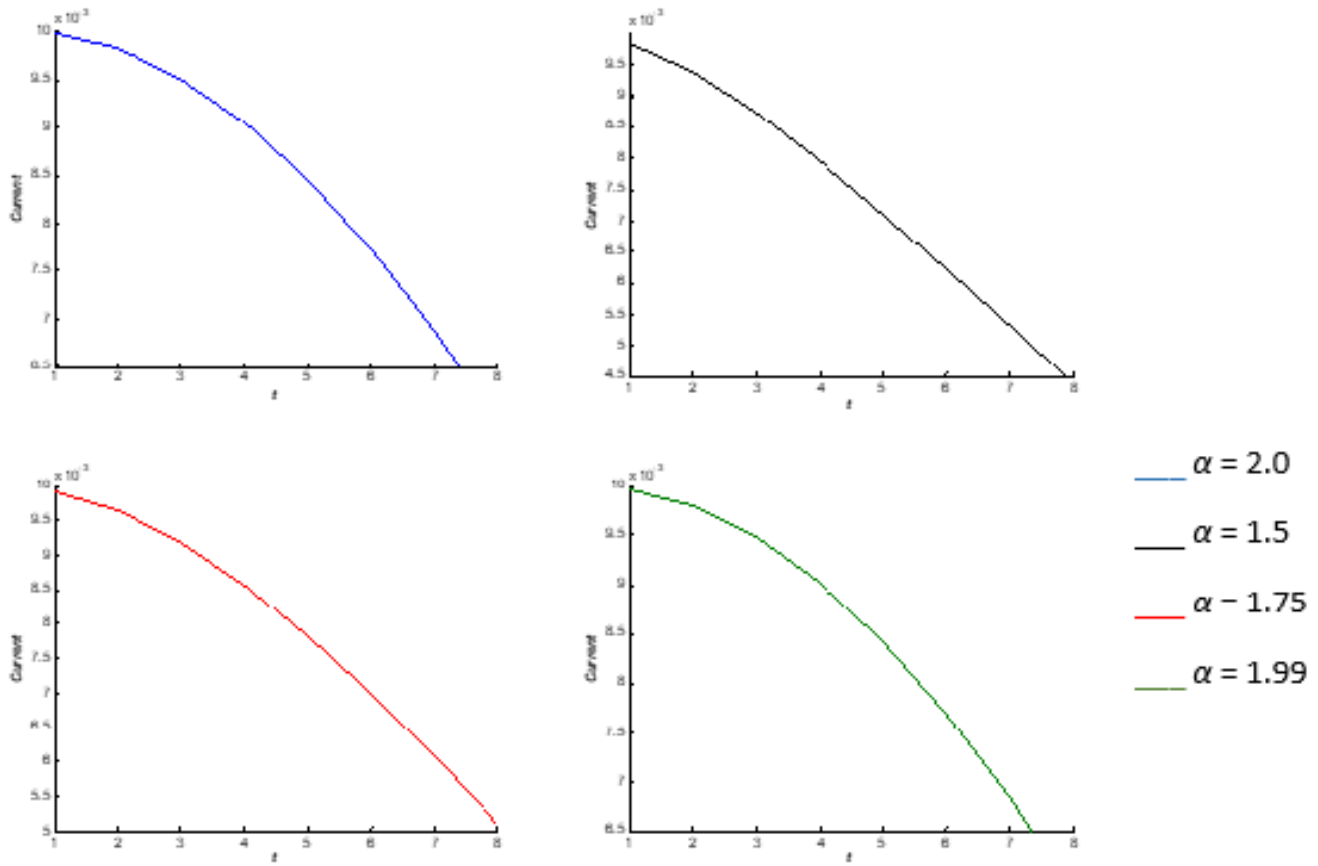

Figure 1: Charge $Q(t)$ vs Time Graph

$\left(L=1, C=1, V_{0}=0.01\right.$ and $\alpha=1.5,1.75,1.99$ and 2$)$

The classical solution of (22) for $\alpha=1$ is

$$
U(t)=U_{0} e^{-\mu t} .
$$

Taking equation (22) and applying the proposed method, let

$$
D^{\alpha} U(t)=a_{m}^{T} H_{m} .
$$

Integrating above equation with respect to $t$, we obtain

$$
U(t)=a_{m}^{T} P^{\alpha} H_{m}+U(0) .
$$


After using the given conditions, we get

$$
U(t)=a_{m}^{T} P^{\alpha} H_{m}+U_{0} .
$$

Now equation (22) will be converted into matrix form as

$$
a_{m}^{T} H_{m}+\mu\left[a_{m}^{T} P^{\alpha} H_{m}+U_{0}\right]=0 .
$$

The above matrix equation can be solved by using MATLAB to find the wavelet coefficients vector $a_{m}^{T}$. The numerical solutions of the RC circuit for some values of $\alpha$ are given in the Table 2. Also graphical analysis for different values of $\alpha=0.5,0.75,1.0$ with $m=8$ is shown in Figure 2. As it can be clearly seen, for $\alpha=1.0$ with $m=8$, the graphical behavior of fractional $\mathrm{RC}$ circuit is similar to the classical solution for $\alpha=1$.

Table 2: Numerical Results of RC Circuit

$\left(R=10, C=1, U_{0}=20\right.$ and $\alpha=0.5,0.75$ and 1.0$)$.

\begin{tabular}{|c|c|c|c|c|}
\hline \multirow{2}{*}{$t$} & $\alpha=0.5$ & $\alpha=0.75$ & $\alpha=1.0$ & $\alpha=1.0$ \\
\cline { 2 - 5 } & HW & HW & HW & CS \\
\hline $1 / 16$ & 19.4819 & 19.7420 & 19.8758 & 19.8754 \\
\hline $3 / 16$ & 19.0637 & 19.3946 & 19.6289 & 19.6285 \\
\hline $5 / 16$ & 18.8005 & 19.1177 & 19.3850 & 19.3847 \\
\hline $7 / 16$ & 18.5919 & 18.8727 & 19.1442 & 19.1439 \\
\hline $9 / 16$ & 18.4147 & 18.6487 & 18.9064 & 18.9061 \\
\hline $11 / 162$ & 18.2588 & 18.4401 & 18.6715 & 18.6712 \\
\hline $13 / 16$ & 18.1184 & 18.2437 & 18.4396 & 18.4394 \\
\hline $15 / 16$ & 17.9900 & 18.0572 & 18.2105 & 18.2102 \\
\hline
\end{tabular}

\section{RLC Circuit}

Consider the fractional RLC circuit with resistance, inductance and charged capacitance. The related differential equation of fractional RLC circuit is

$$
D^{2 \alpha} Q(t)+\rho Q^{\alpha}(t)+\kappa Q(t)=0, \quad \text { where } \quad 0 \leq \alpha \leq 1
$$

with conditions $Q(0)=Q_{0}$ and $D^{\alpha} Q(0)=0$ where $\kappa=\frac{1}{L C}$ and $\rho=\frac{R}{L}$.

The classical solution of (28) for $\alpha=1$ is

$$
Q(t)=Q_{0} e^{\frac{-\rho}{2} t} \cos \left(\sqrt{\kappa-\frac{\rho^{2}}{4}} t\right) .
$$

Applying Haar wavelet method to equation (28), let

$$
D^{2 \alpha} Q(t)=a_{m}^{T} H_{m}
$$



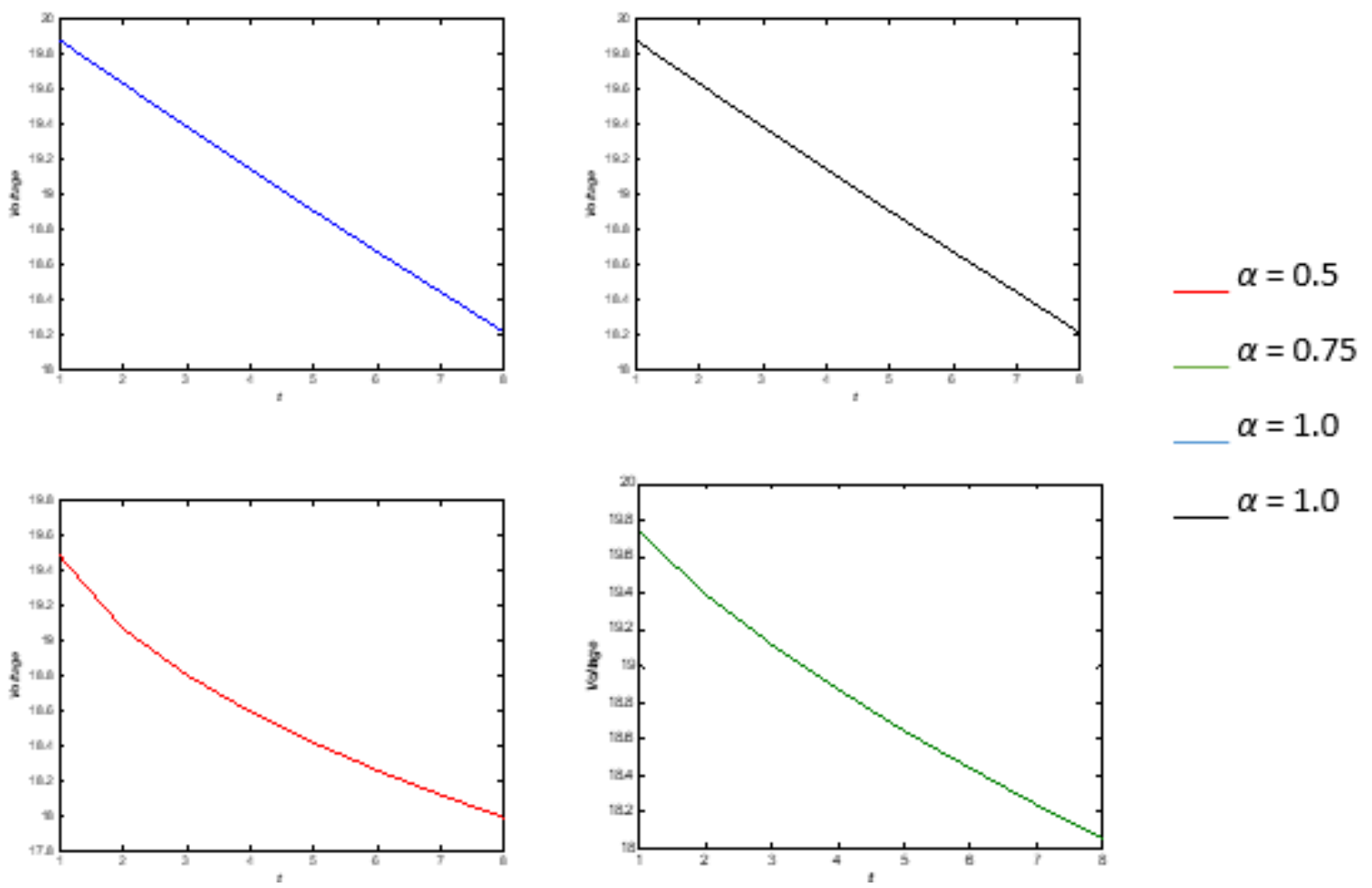

$\alpha=1.0$

Figure 2: Voltage vs Time

$\left(R=10, C=1, U_{0}=20\right.$ and $\alpha=0.5,0.75$ and 1.0$)$

Integrating above Equation (30) with respect to $t$, we get

$$
D^{\alpha} Q(t)=a_{m}^{T} P^{\alpha} H_{m}+Q^{\alpha}(0)
$$

Integrating Equation (31) again with respect to $t$, we obtain

$$
Q(t)=a_{m}^{T} P^{2 \alpha} H_{m}+t Q^{\alpha}(0)+Q(0) .
$$

After using the conditions in equation (32), we get

$$
Q(t)=a_{m}^{T} P^{2 \alpha} H_{m}+Q_{0}
$$

Now equation (28) will be converted into matrix form as

$$
a_{m}^{T} H_{m}+\rho\left[a_{m}^{T} P^{\alpha} H_{m}\right]+\kappa\left[a_{m}^{T} P^{2 \alpha} H_{m}+Q_{0}\right]=0
$$

The matrix equation is solved by using MATLAB to find the wavelet coefficients vector $a_{m}^{T}$. The numerical solutions ofthe RLC circuit for different value of $\alpha$ are given in the Table 3. Also graphical analysis for different values of $\alpha=0.75,0.95,1.0$ with $m=8$ is shown in Figure 3 . As can be seen, for $\alpha=1.0$ with $m=8$, the graphical behavior of fractional RLC circuit is close to the classical solution for $\alpha=1$. 
Table 3: Numerical Results of RLC Circuit

$\left(R=10, C=10, L=20, Q_{0}=20\right.$ and $\alpha=0.75,0.95$ and 1.0$)$.

\begin{tabular}{|c|c|c|c|c|}
\hline \multirow{2}{*}{$\mathrm{t}$} & $\alpha=0.75$ & $\alpha=0.95$ & $\alpha=1.0$ & $\alpha=1.0$ \\
\cline { 2 - 5 } & $\mathrm{HW}$ & $\mathrm{HW}$ & $\mathrm{HW}$ & $\mathrm{CS}$ \\
\hline $1 / 16$ & $9.8 \times 10^{-3}$ & $9.8 \times 10^{-3}$ & $9.8 \times 10^{-3}$ & $9.8 \times 10^{-3}$ \\
\hline $3 / 16$ & $9.5 \times 10^{-3}$ & $9.5 \times 10^{-3}$ & $9.5 \times 10^{-3}$ & $9.5 \times 10^{-3}$ \\
\hline $5 / 16$ & $9.2 \times 10^{-3}$ & $9.2 \times 10^{-3}$ & $9.2 \times 10^{-3}$ & $9.2 \times 10^{-3}$ \\
\hline $7 / 16$ & $8.9 \times 10^{-3}$ & $8.9 \times 10^{-3}$ & $8.9 \times 10^{-3}$ & $8.9 \times 10^{-3}$ \\
\hline $9 / 16$ & $8.6 \times 10^{-3}$ & $8.6 \times 10^{-3}$ & $8.6 \times 10^{-3}$ & $8.6 \times 10^{-3}$ \\
\hline $11 / 16$ & $8.3 \times 10^{-3}$ & $8.3 \times 10^{-3}$ & $8.3 \times 10^{-3}$ & $8.3 \times 10^{-3}$ \\
\hline $13 / 16$ & $8.1 \times 10^{-3}$ & $8.1 \times 10^{-3}$ & $8.1 \times 10^{-3}$ & $8.0 \times 10^{-3}$ \\
\hline $15 / 16$ & $7.8 \times 10^{-3}$ & $7.8 \times 10^{-3}$ & $7.8 \times 10^{-3}$ & $7.7 \times 10^{-3}$ \\
\hline
\end{tabular}
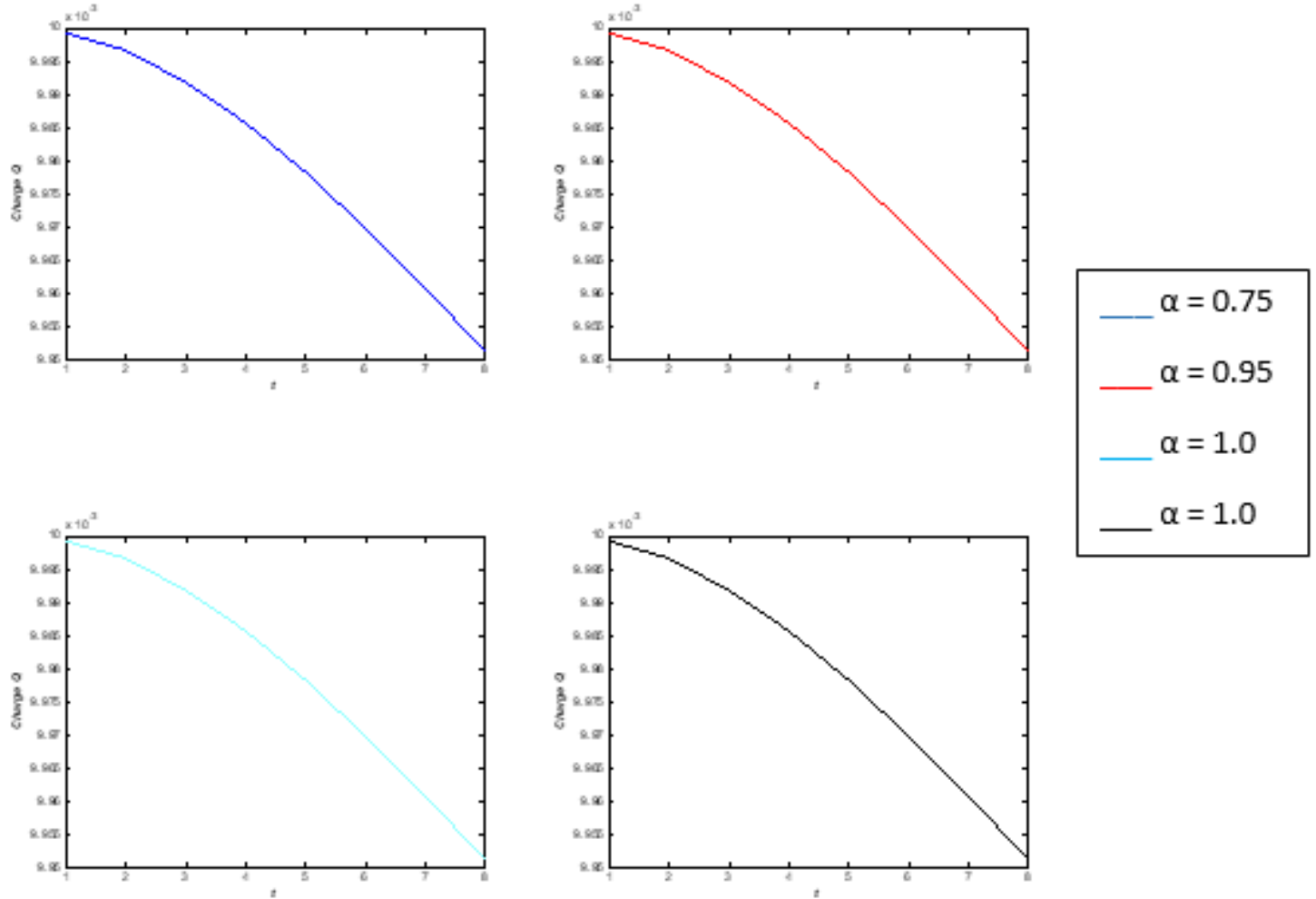

Figure 3: Charge $Q(t)$ vs Time Graph

$$
\left(L=10, R=10, C=10, Q_{0}=0.01 \text { and } \alpha=0.75,0.95 \text { and } 1.0\right)
$$

\section{Conclusion}

The paper proposed Haar's wavelet method, which is more efficient and simple compared to the contemporary solutions available currently used to approximate solutions of the fractional 
electrical circuit models. We have applied our method on three different circuits, namely, LC, $\mathrm{RC}$ and RLC shown in the Tables $(1-3)$, respectively. The tables depicts the relationship of the parameter alpha. As alpha increases, the numerical values approach to the classical solutions for $\alpha=1$. Moreover, on increasing the level of wavelet i.e for $m=16$ or $m=32$, we can get more precise solutions of these fractional models. The graphs in Figures $(1-3)$, represents numerical solutions of the fractional models that behave similar to the classical solutions but less complicated and more efficient. It can be concluded that the proposed method gives fast convergence to the solutions, easy in execution and can be applied to other physical models used in real life problems.

\section{Acknowledgements}

Authors are very grateful to the referees for the constructive comments and suggestions for the improvement of the paper.

\section{References}

[1] Oldham, K. and Spanier, J. The Fractional Calculus Theory and Applications of Differentiation and Integration to Arbitrary Order. Elsevier. 1974.

[2] Miller, K. S. and Ross, B. An Introduction to the Fractional Calculus and Fractional Differential Equations. New York: Wiley. 1993.

[3] Podlubny, I. Fractional Differential Equations: An Introduction to Fractional Derivatives, Fractional Differential Equations, to Methods of Their Solution and Some of Their Applications. Elsevier. 1998.

[4] Abbas, S., Benchohra, M. and N'Guérékata, G. M. Topics in Fractional Differential Equations. Springer Science and Business Media. 2012.

[5] Diethelm, K. The Analysis of Fractional Differential Equations: An Application-oriented Exposition using Differential Operators of Caputo Type. Springer Science and Business Media. 2010.

[6] Prakash, A., Goyal, M., \& Gupta, S. Fractional variational iteration method for solving time-fractional Newell-Whitehead-Segel equation. Nonlinear Engineering. 2019. 8(1): 164171.

[7] Akgül, E. K. Solutions of the linear and nonlinear differential equations within the generalized fractional derivatives.Chaos: An Interdisciplinary Journal of Nonlinear Science. 2019. 29(2). 023108. https://doi.org/10.1063/1.5084035.

[8] Shen, S., Liu, F., and Anh, V. V. The analytical solution and numerical solutions for a two-dimensional multi-term time fractional diffusion and diffusion-wave equation. Journal of Computational and Applied Mathematics. 2019. 345. 515-534.

[9] Karamali, G., Dehghan, M., and Abbaszadeh, M. Numerical solution of a time-fractional PDE in the electroanalytical chemistry by a local meshless method. Engineering with Computers. 2019. 35(1): 87-100. 
[10] Javeed, S., Baleanu, D., Waheed, A., Shaukat Khan, M., and Affan, H. Analysis of homotopy perturbation method for solving fractional order differential equations. Mathematics. 2019. 7(1) 40. https://doi.org/10.3390/math7010040.

[11] Demir, A., Bayrak, M. A. and Ozbilge, E. A New Approach for the Approximate Analytical Solution of Space-Time Fractional Differential Equations by the Homotopy Analysis Method. Advances in Mathematical Physics. 2019. Article ID 5602565, 12 pages, 2019. https://doi.org/10.1155/2019/5602565.

[12] Kundu, S. Analytical solutions of one-dimensional space-fractional advection-diffusion equation for sediment suspension using homotopy analysis method. Journal of Engineering Mechanics. 2019. 145(2). 04019048. https://doi.org/10.1061/(ASCE)EM. 1943-7889.0001625.

[13] Haq, S., Ghafoor, A., Hussain, M., and Arifeen, S. Numerical solutions of two dimensional Sobolev and generalized Benjamin-Bona-Mahony-Burgers equations via Haar wavelets. Computers and Mathematics with Applications. 2019. 77(2): 565-575.

[14] Oruç, Ö. A non-uniform Haar wavelet method for numerically solving twodimensional convection-dominated equations and two-dimensional near singular elliptic equations. Computers \&5 Mathematics with Applications. 2019. 77(2): 1799-1820.

[15] Singh, I., and Kumar, S. Haar wavelet methods for numerical solutions of Harry Dym (HD), BBM Burger's and 2D diffusion equations. Bulletin of the Brazilian Mathematical Society, New Series. 2018. 1-26.

[16] Yuttanan, B., and Razzaghi, M. Legendre wavelets approach for numerical solutions of distributed order fractional differential equations. Applied Mathematical Modelling. 2019. 70: $350-364$.

[17] Moradi, L., Mohammadi, F., and Baleanu, D. A direct numerical solution of time-delay fractional optimal control problems by using Chelyshkov wavelets. Journal of Vibration and Control. 2019. 25(2): 310-324.

[18] Haar, A. Zur Theorie der orthogonalen Funktionensysteme. Mathematische Annalen. 1910. 69: 331-371.

[19] Chen, C. F., and Hsiao, C. H. Haar wavelet method for solving lumped and distributedparameter systems. IEEE Proceedings-Control Theory and Applications. 1997. 144(1): 8794.

[20] Khashan, M. M., Amin, R., and Syam, M. I. A new algorithm for fractional Riccati type differential equations by using Haar wavelet. Mathematics. 2019. 7(6): 545. MDPI AG. http://dx.doi.org/10.3390/math7060545

[21] Xie, J., Wang, T., Ren, Z., Zhang, J., and Quan, L. Haar wavelet method for approximating the solution of a coupled system of fractional-order integraldifferential equations. Mathematics and Computers in Simulation. 2019. 163, 80-89. https://doi.org/10.1016/j.matcom.2019.02.010.

[22] Oruç, Ö., Esen, A., \& Bulut, F. A Haar wavelet approximation for two-dimensional time fractional reaction-subdiffusion equation. Engineering with Computers. 2019. 35(1). 75-86. 
[23] Gómez, J. F., Astorga, C. M., Escobar, R. F., Medina, M. A., Guzmán, R., Gonzáleg, A., and Baleanu, D. Overview of simulation of fractional differential equation using numerical Laplace transform. Cent. Eur. J. Phys. (CEJP). 2012. 1-14.

[24] Shah, P. V., Patel, A. D., Salehbhai, I. A., and Shukla, A. K. Analytic solution for the electric circuit model in fractional order. Abstract and Applied Analysis. Hindawi. 2014. Hindawi. http://dx.doi.org/10.1155/2014/343814.

[25] Alsaedi, A., Nieto, J. J., and Venktesh, V. Fractional electrical circuits. Advances in Mechanical Engineering. 2015. 7(11). https://doi.org/10.1177/1687814015618127.

[26] Gill, V., Modi, K., and Singh, Y. Analytic solutions of fractional differential equation associated with RLC electrical circuit. Journal of Statistics and Management Systems. 2018. 21(4): 575-582.

[27] Arora, R., and Chauhan, N. S. An application of Legendre wavelet in fractional electrical circuits. Global Journal of Pure and Applied Mathematics. 2017. 13(2): 183-202.

[28] Atangana, A., and Nieto, J. J. Numerical solution for the model of RLC circuit via the fractional derivative without singular kernel. Advances in Mechanical Engineering. 2015. 7(5): 1687814015613758.

[29] Gómez-Aguilar, J. F., Yépez-Martínez, H., Escobar-Jiménez, R. F., Astorga-Zaragoza, C. M., and Reyes-Reyes, J. Analytical and numerical solutions of electrical circuits described by fractional derivatives. Applied Mathematical Modelling. 2016. 40(21-22): 9079-9094.

[30] Sene, N., and Gómez-Aguilar, J. F. Analytical solutions of electrical circuits considering certain generalized fractional derivatives. The European Physical Journal Plus. 2019. 134(6). 260. https://doi.org/10.1140/epjp/i2019-12618-x.

[31] Li, Y., and Zhao, W. Haar wavelet operational matrix of fractional order integration and its applications in solving the fractional order differential equations. Applied Mathematics and Computation. 2010. 216(3): 2276-2285.

[32] Kilicman, A., and Al Zhour, Z. A. A. Kronecker operational matrices for fractional calculus and some applications. Applied Mathematics and Computation. 2007. 187(1): 250-265. 